\title{
The Impact of Nimodipine Administration through Feeding Tube on Outcomes in Patients with Aneurysmal Subarachnoid Hemorrhage
}

\author{
Fadumo Ahmed Isse, Yasmeen El Hajj Abdallah and Sherif Hanafy Mahmoud. \\ Faculty of Pharmacy and Pharmaceutical Sciences, University of Alberta, Edmonton, AB, Canada. \\ Received, February 24, 2020; Revised, April 18, 2020; Accepted, April 19, 2020; Published, April 21, 2020.
}

\begin{abstract}
PURPOSE: Delayed cerebral ischemia (DCI) and vasospasm are the main challenges contributing to unfavorable outcomes following aneurysmal subarachnoid hemorrhage. Nimodipine has been shown to decrease the incidence of delayed cerebral ischemia and improve outcomes. In patients who are unable to swallow, nimodipine tablets are crushed and administered through enteral feeding tubes. However, it is not clear whether this may result in reduced clinical effectiveness. The aims of the study were to investigate the impact of nimodipine administration through enteral feeding tubes, in the first 7 days and over the 21-days period on patient outcomes. METHODS: A retrospective chart review of subarachnoid hemorrhage patients admitted at the University of Alberta Hospital, Edmonton, Alberta, Canada was carried out. Logistic regression modelling was utilized to identify predictors of vasospasm and delayed cerebral ischemia. Main outcome measures were angiographic evidence of moderate to severe vasospasm, development of delayed cerebral ischemia and hospital mortality. RESULTS: 85 patients were included. Following adjustment for disease severity, nimodipine administration technique was associated with vasospasm in the first 7 days of patient admission where patients receiving nimodipine via enteral feeding tubes had increased odds of vasospasm compared to those administered it as whole tablets (OR 8.9,95\% CI 1.1-73.1, $p$ value 0.042 ). When analyzed over the 21-day period, nimodipine administration by feeding tube was associated with increased odds of DCI compared to whole tablets (OR 38.1, 95\% CI 1.4-1067.9, $p$ value 0.032). CONCLUSIONS: Our findings suggest that nimodipine administration via enteral feeding tubes may be associated with vasospasm and DCI in subarachnoid hemorrhage patients secondary to reduced exposure. Prospective studies are needed to confirm such association and alternate methods of administration should be explored to ensure patients are getting the benefits of nimodipine.
\end{abstract}

\section{INTRODUCTION}

Aneurysmal subarachnoid hemorrhage (SAH) is a life-threatening condition characterized by the extravasation of blood into the subarachnoid space secondary to a ruptured intracranial aneurysm. Although SAH accounts for 5-10\% of all strokes, it affects patients at a relatively young age leading to premature loss of productive life. Mortality rates secondary to SAH has been reported to range from $30-50 \%$, leaving the rest of patients with different degrees of disability (1-3). Although there are various medical and neurological complications following $\mathrm{SAH}$, delayed cerebral ischemia (DCI) and vasospasm are the main challenges that contribute to unfavorable patient outcomes. Effective prevention of DCI can significantly improve the functional outcomes of patients $(4,5)$. The role of nimodipine, a dihydropyridine calcium channel blocker with selectivity towards the cerebral blood vessels, in preventing DCI has been investigated by several randomized clinical trials. It has been shown to decrease the incidence of DCI and improve patient outcomes (6-9). Therefore, the current guidelines suggests that all patients who are admitted for SAH to receive nimodipine for 21 days orally and to be started within 96 hours from ictus (1). In our institution, all patients presenting with SAH receive oral nimodipine $60 \mathrm{mg}$ every 4 hours for 21 days. Nimodipine oral tablet is the only available formulation in Canada. Therefore, patients will swallow the whole tablets if they are able to, otherwise, nimodipine tablets are crushed at bedside, suspended in water and administered immediately through enteral feeding tubes (FT) for those who are unable to swallow, such as those with altered mental

Corresponding Author: Sherif Hanafy Mahmoud, BSc (Pharm), MSc., PhD; ORCID number: https://orcid.org/00000002-5517-2622; Faculty of Pharmacy and Pharmaceutical Sciences, University of Alberta, 3-142H Katz Group Centre for Pharmacy and Health Research, Edmonton, AB, Canada, Email: smahmoud@ualberta.ca 
status or mechanically ventilated. However, nimodipine drug product monograph states that the tablet should not be crushed as it may result in reduced drug bioavailability (11). In addition, few small studies have reported reduced bioavailability of nimodipine administered through FT and in those with high grade SAH $(12,13)$. It is not clear, however, whether this technique of administration result in reduced clinical effectiveness and in turn poor outcomes. Although the evidence supporting a correlation between nimodipine plasma concentrations and patient outcomes is scarce and not clear, lack of absorption definitely denies the benefits of any drug used systemically (14). Therefore, the present study aimed to answer the following research question: Among SAH patients, is taking nimodipine by enteral feeding tube associated with worse outcomes compared to those who swallow nimodipine whole tablets?

The primary aim of the current study was to investigate the impact of nimodipine administration through enteral feeding tubes, in the first 7 days from onset, on the outcomes in patients with SAH. We chose the first 7 days of exposure as the onset of vasospasm and DCI are generally within that time period. In addition, the secondary aim was to determine the impact of nimodipine mode of administration throughout the whole course (21 days), on the outcomes in patients with SAH. To our knowledge, the present study is the first to investigate the association between nimodipine techniques of oral administration with patient outcomes.

\section{METHODS}

\section{Study Design}

A retrospective chart review of adult patients diagnosed with SAH and admitted to the University of Alberta Hospital, Edmonton, Canada from January 2016 to December 2018. The study was approved by the Health Research Ethics Board (HERB) of the University of Alberta and have been performed in accordance with the ethical standards as laid down in the 1964 Declaration of Helsinki and its later amendments or comparable ethical standards. For this type of study, formal consent is not required.

\section{Study Population}

Patient medical records (paper and electronic) were requested based on ICD-10-CA codes for SAH. The codes included were: I60.0 Subarachnoid hemorrhage from carotid siphon and bifurcation, I60.1 Subarachnoid hemorrhage from middle cerebral artery, I60.2 Subarachnoid hemorrhage from anterior communicating artery, I60.3 Subarachnoid hemorrhage from posterior communicating artery, I60.4 Subarachnoid hemorrhage from basilar artery, I60.5 Subarachnoid hemorrhage from vertebral artery, I60.6 Subarachnoid hemorrhage from other intracranial arteries, and I60.7 Subarachnoid hemorrhage from intracranial artery, unspecified. The inclusion criteria were adult patients (age $\geq 18$ years) admitted to the University of Alberta Hospital Neurosciences intensive care unit (ICU) with aneurysmal subarachnoid hemorrhage diagnosis and were treated with nimodipine regardless of the mode of administration. The exclusion criteria were the following: non-aneurysmal $\mathrm{SAH}$, delayed presentation and those who were treated with nimodipine for less than 4 days.

\section{Data Extraction}

Patients' charts were reviewed and data were collected and managed using REDCap database capture tool hosted at the University of Alberta (15). Patients' demographics (age, sex, height, weight and body mass index (BMI)), past medical history (history of diabetes, hypertension, kidney and liver disease) and social history (smoking and alcohol intake) were recorded. In addition, the following baseline data were included: admission Glasgow coma scale (GCS), World Federation of Neurological Surgeons (WFNS) grade, Fisher scale, Acute Physiology and Chronic Health Evaluation (APACHE II) score, aneurysm location, and aneurysm intervention (surgical clipping, endovascular coiling or other). APACHE II physiological subscore was determined by subtracting admission GCS and age from APACHE II score.

Nimodipine administration record including dose, frequency, duration, method of administration (swallowed whole tablets (PO) vs being crushed and administered by enteral feeding tube (FT)) and any missed doses were recorded. In addition, administration of interacting drugs (cytochrome P450 enzyme inducers and inhibitors) were collected. The median percentage of nimodipine administration was calculated by dividing the number of treatment days by the hospital length of stay in the first week. In addition, for the 21-days full 
period, the percentage was calculated by dividing the number of days of nimodipine treatment by 21 days or hospital length of stay (if shorter than 21 days). Primary outcomes collected included angiographic evidence of moderate to severe vasospasm (diagnosed through digital subtraction angiography), development of DCI and hospital mortality. DCI was defined as the documentation of new onset focal neurological impairment (such as hemiparesis or aphasia), cerebral infarction or a decrease of at least 2 points in GCS and cannot be explained by other causes (16). In addition, hospital and ICU length of stay, need for external ventricular drain (EVD), rebleeding and discharge disposition were recorded.

\section{DATA ANALYSIS}

Patients' baseline characteristics and the outcomes were summarized. Continuous variables that are normally distributed were presented as mean \pm standard deviation and compared by Student's t test; otherwise, they were presented as median with interquartile range (IQR) and compared by Wilcoxon rank sum test. Categorical variables were displayed as frequency and percentage, $\mathrm{n}(\%)$ and compared by $\chi^{2}$ or Fisher exact test, as appropriate. For the primary aim, patients who took nimodipine by enteral feeding tubes $\geq 4$ days in the first week were classified as feeding tube group (FT7) and for those who were administered nimodipine as whole tablets $\geq 4$ days in the first week were classified as oral group (PO7). For the secondary aim, patients who were administered nimodipine as whole tablets $\geq 50 \%$ of the duration of therapy (up to 21-days) were considered as oral group (PO21). Otherwise, they were included in the feeding tube group (FT21). The association between individual covariates (such as nimodipine administration by feeding tube, baseline characteristics and disease severity) and primary outcomes were determined using univariate logistic regression. Variables with estimated $p$ value of $<0.2$ or biologically plausible were included in multivariate logistic regression models and adjusted odds ratios (OR) were determined. The fit of the final model was confirmed by using Hosmer-Lemeshow goodness-of-fit test. Area under the receiver operating characteristic (ROC) curve was determined to confirm model discrimination. Missing data, if any, were handled by complete case analysis. Level of significance was set at $p$ value $<$ 0.05. STATA software version 15 (STATA Corp, College station, Texas) was used for data analysis.

\section{RESULTS}

\section{Study Participants}

Medical records of 134 patients were identified. A total of 49 patients were excluded as they did not meet the inclusion criteria (Figure 1). As a result, 85 patients were included in the present study.

\section{Baseline Characteristics}

Among the 85 included patients, 60 took nimodipine as whole tablets (PO7) and 25 got the drug via feeding tube (FT7) in the first week. Patients' baseline characteristics are presented in Table 1. Generally, both groups were comparable with regards to their demographics, past medical and social history. The mean ages in the FT7 and PO7 groups were $52 \pm 11$ and $55 \pm 14$ years, respectively and approximately two-thirds of the patients were females. On the other hand, as expected, patients in the FT7 group were generally sicker compared to the PO7 group. They had lower GCS on admission [8 (IQR 8) vs 15 (IQR 1 ), $p$ value $<0.001$ ], and higher WFNS grade ( $p$ value $<0.001$ ), Fisher Scale ( $p$ value 0.009 ) and APACHE II subscore ( $p$ value 0.0008 ) compared to PO7 group. Besides, more patients got their aneurysm treated with endovascular coiling in the PO7 group compared to FT7 group ( $p$ value 0.049 ). None of the patients included in the current study had history of liver or kidney disease.

Most of patients were treated with nimodipine at a dose of $60 \mathrm{mg}$ every four hours; however, 4 patients in the PO7 and 5 patients in the FT7 groups were switched to $30 \mathrm{mg}$ every 2 hours in the first week of nimodipine administration due to reduced blood pressure. Median duration and percentage of nimodipine administration are shown in Table 2. Both groups had comparable percentages of nimodipine treatment throughout the hospital stay.

\section{Hospital course and clinical outcomes}

Out of the 85 patients participated in the study, 8 (9\%) patients died and 21 (25\%) developed vasospasm (15 patients had angiographic vasospasm; 6 had their vasospasm documented by the managing team in progress notes but their angiography reports were not available). Six patients fitted the criteria of DCI: aphasia and weakness $(n=1)$, right sided weakness $(n=1)$, left sided weakness $(n=1)$, cerebral infarction $(n=2)$ and neurological deterioration $(n=1)$. 


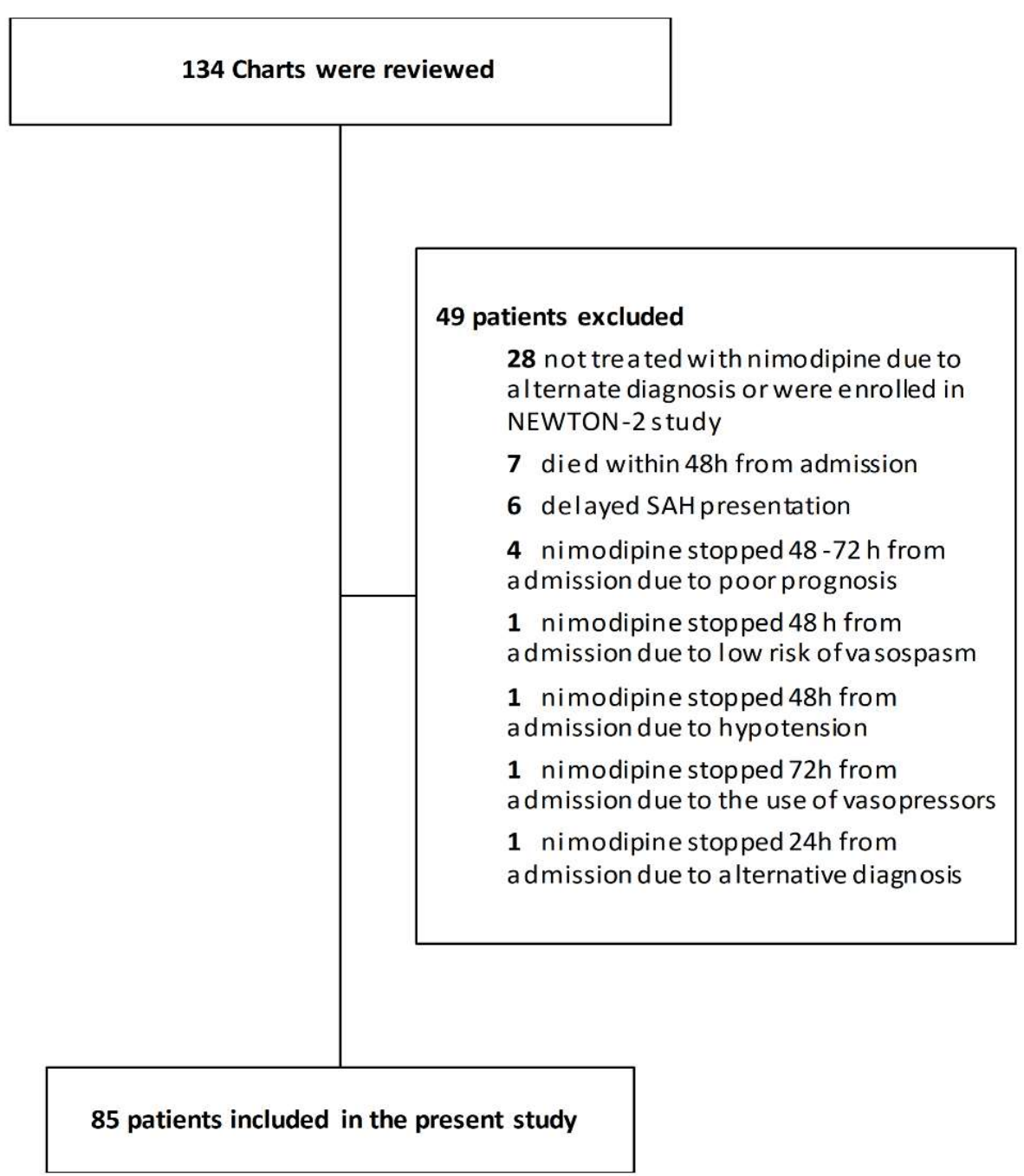

Figure 1. Flow chart of patients included in the present study

All of those who developed DCI had moderate to severe angiographic vasospasm. The median onset days for angiographic vasospasm and DCI were 7 (IQR 2) and 7 (IQR 4), respectively. The median ICU and hospital length of stay were 12 (IQR 11) and 18 (IQR 25), respectively. A total of 47 (55\%) patients needed external ventricular drain insertion throughout their stay. With regards to discharge disposition, $50(59 \%)$ patients were discharged home with or without support services and 27 (32\%) were transferred to another acute care or continuing care facilities.

\section{The impact of nimodipine administration through enteral feeding tube in the first week on patient outcomes}

The impact of nimodipine administration techniques on angiographic vasospasm, DCI and hospital mortality was tested using univariate and multivariate logistic regression models. Table 3 depicts the crude and adjusted odds ratios for predictors of angiographic vasospasm in the best-fit logistic regression model. As shown in the table, after adjusting for disease severity (WFNS, Fisher and APACHE II subscore) and aneurysm intervention, nimodipine administration technique was associated with vasospasm in the first 7 days of patient admission where patients receiving nimodipine via FT had increased odds of angiographic vasospasm compared to those administered it as whole tablets (OR 8.9, 95\% CI 1.173.1, $p$ value 0.042 ; ROC AUC 0.87, HL-test not significant). In addition, we found that APACHE subscore $>15$ is also an independent predictor for angiographic vasospasm. 
DCI was observed only in patients with Fisher scale III and IV. Therefore, to control for Fisher scale as a confounder in the DCI regression model we analyzed only those with Fisher scale of III and IV $(n=58)$. Administering nimodipine technique was not associated with DCI (OR 18 for TF compared to
PO, 95\% CI 0.6-570.5, $p$ value 0.102; ROC AUC 0.92, HL-test not significant). Furthermore, nimodipine administration technique was not significantly associated with mortality ( $p$ value $0.087)$.

Table 1. Baseline characteristics of patients included in the study

\begin{tabular}{|c|c|c|c|}
\hline & $\begin{array}{l}\text { PO7 } \\
(n=60)\end{array}$ & $\begin{array}{l}\text { FT7 } \\
(\mathrm{n}=\mathbf{2 5})\end{array}$ & $p$ value \\
\hline Age, mean \pm SD & $55 \pm 14$ & $52 \pm 11$ & 0.387 \\
\hline Female sex, n (\%) & $40(67)$ & $14(56)$ & 0.352 \\
\hline Height $(\mathrm{cm})$, mean $\pm \mathrm{SD}^{1}$ & $166 \pm 9$ & $172 \pm 8$ & 0.015 \\
\hline Weight $(\mathrm{kg})$, mean $\pm \mathrm{SD}$ & $78 \pm 20$ & $81 \pm 26$ & 0.632 \\
\hline BMI, mean $\pm \mathrm{SD}^{1}$ & $28 \pm 7$ & $27 \pm 8$ & 0.485 \\
\hline BMI categories, $\mathrm{n}(\%)^{1}$ & & & 0.195 \\
\hline $17-24.9 \mathrm{~kg} / \mathrm{m}^{2}$ & $22(37)$ & $11(46)$ & \\
\hline $25-29.9 \mathrm{~kg} / \mathrm{m}^{2}$ & $19(32)$ & $10(42)$ & \\
\hline$\geq 30 \mathrm{~kg} / \mathrm{m}^{2}$ & $19(32)$ & $3(13)$ & \\
\hline \multicolumn{4}{|l|}{ Medical history } \\
\hline Hypertension, $\mathrm{n}(\%)$ & $23(38)$ & $5(20)$ & 0.101 \\
\hline Diabetes, $\mathrm{n}(\%)$ & $7(12)$ & $3(12)$ & 1.000 \\
\hline \multicolumn{4}{|l|}{ Smoking history, n (\%) } \\
\hline Smoker/Ex-smoker & $28(47)$ & $14(56)$ & 0.480 \\
\hline Non-smoker & $14(23)$ & $3(12)$ & \\
\hline Unknown & $18(30)$ & $8(32)$ & \\
\hline \multicolumn{4}{|l|}{ Alcohol history, n (\%) } \\
\hline Heavy drinker & $8(13)$ & $5(20)$ & 0.512 \\
\hline Others & $52(87)$ & $20(80)$ & \\
\hline \multicolumn{4}{|l|}{ SAH characteristics, n (\%) } \\
\hline Location & & & 0.101 \\
\hline MCA & $9(15)$ & $8(32)$ & \\
\hline ACOMM & $30(50)$ & $6(24)$ & \\
\hline PCOM & $7(12)$ & $5(20)$ & \\
\hline Others & $14(23)$ & $6(24)$ & \\
\hline Intervention & & & 0.049 \\
\hline Coil & $42(70)$ & $11(44)$ & \\
\hline Clip & $17(28)$ & $12(48)$ & \\
\hline Others & $1(2)$ & $2(8)$ & \\
\hline Fisher Scale ${ }^{2}$ & & & 0.009 \\
\hline I-II & $24(41)$ & $3(12)$ & \\
\hline III-IV & $34(59)$ & $22(88)$ & \\
\hline WFNS grade & & & $<0.001$ \\
\hline Grade I-III & $55(92)$ & $8(32)$ & \\
\hline Grade IV-V & $5(8)$ & $17(68)$ & \\
\hline Admission GCS, median $\pm \mathrm{IQR}$ & $15 \pm 1$ & $8 \pm 8$ & $<0.001$ \\
\hline APACHE, mean \pm SD & $12 \pm 4$ & $20 \pm 5$ & $<0.001$ \\
\hline APACHE sub score, mean \pm SD & $8 \pm 3$ & $12 \pm 5$ & 0.0008 \\
\hline CYP enzyme inducer, n (\%) & $2(3)$ & $4(16)$ & 0.059 \\
\hline \multicolumn{4}{|c|}{$\begin{array}{l}\text { ACOMM, anterior communicating artery; APACHE, Acute Physiology and Chronic Health Evaluation; BMI, body mass } \\
\text { index; CYP, cytochrome P450; FT7, patients who took nimodipine by feeding tube (crushed) in the first week; GCS, } \\
\text { Glasgow Coma Scale; MCA, middle cerebral artery; PO7, patients who took nimodipine orally as whole tablets in the first } \\
\text { week; PCOM, posterior communicating artery; WFNS, World Federation of Neurological Surgeons. }{ }^{1},(\text { FT7, } n=24){ }^{2} \text {, } \\
\text { (PO7, } n=58 \text { ). }\end{array}$} \\
\hline
\end{tabular}


Table 2. Median duration and percentage of nimodipine administration in the present study

\begin{tabular}{|c|c|c|c|c|c|c|}
\hline & \multicolumn{3}{|c|}{ First 7 days or until discharge (if $<7$ d) } & \multicolumn{3}{|c|}{ Over 21 days or until discharge (if $<21$ d) } \\
\hline & $\mathrm{PO} 7(n=60)$ & FT7 $(n=25)$ & $p$ value & $\mathrm{PO} 21(n=64)$ & FT21 $(n=21)$ & $p$ value \\
\hline $\begin{array}{l}\text { No. of days of nimodipine } \\
\text { administration }\end{array}$ & $7(1)$ & $6(1)$ & 0.234 & $12(11)$ & $16(12)$ & 0.455 \\
\hline $\begin{array}{l}\text { \% of nimodipine } \\
\text { administration overall }\end{array}$ & $100(14)$ & $86(14)$ & 0.429 & $89(12)$ & $90(24)$ & 0.653 \\
\hline $\begin{array}{l}\text { \% of nimodipine } \\
\text { administration by } \text { FT }^{2}\end{array}$ & $0(0)$ & $100(25)$ & $<0.001$ & $100(7)$ & $0(15)$ & $<0.001$ \\
\hline $\begin{array}{l}\text { \% of nimodipine } \\
\text { administration by } \mathrm{PO}^{2}\end{array}$ & $100(0)$ & $0(25)$ & $<0.001$ & $0(7)$ & $100(15)$ & $<0.001$ \\
\hline \multicolumn{7}{|c|}{$\begin{array}{l}\text { Data presented as median (IQR). }{ }^{1} \text { The median percentage of nimodipine administration was calculated by dividing the } \\
\text { number of treatment days by the hospital length of stay in the first week. In addition, for the } 21 \text {-days full period, the } \\
\text { percentage was calculated by dividing the number of days of nimodipine treatment by } 21 \text { days or hospital length of stay (if } \\
\text { shorter than } 21 \text { days). }{ }^{2} \text { The percentage of nimodipine administration by feeding tube (FT) or orally as whole tables (PO) } \\
\text { was calculated by dividing the number of days the patient was given nimodipine by FT or PO, respectively, by the total } \\
\text { number of days of nimodipine administration. }\end{array}$} \\
\hline
\end{tabular}

Table 3. Crude and adjusted odds ratios of variables included in the final multivariate logistic regression model of angiographic vasospasm in the first week

\begin{tabular}{|c|c|c|c|c|c|c|}
\hline & \multicolumn{3}{|c|}{ Univariate Regression } & \multicolumn{3}{|c|}{ Multivariate Regression } \\
\hline & OR & $p$ value & $95 \% \mathrm{CI}$ & OR & $p$ value & $95 \% \mathrm{CI}$ \\
\hline Nimodipine (FT7) ${ }^{1}$ & 6.5 & 0.002 & $1.9-21.8$ & 8.9 & 0.042 & $1.1-73.1$ \\
\hline Heavy drinker ${ }^{2}$ & 2.5 & 0.179 & $0.7-9.9$ & 2.0 & 0.515 & $0.3-14.9$ \\
\hline \multicolumn{7}{|l|}{ Location $^{3}$} \\
\hline MCA & Ref & & & & & \\
\hline ACOMM & 0.8 & 0.808 & $0.2-3.4$ & 4.7 & 0.224 & $0.4-57.2$ \\
\hline PCOM & 1.1 & 0.895 & $0.2-6.4$ & 2.6 & 0.464 & $0.2-34.2$ \\
\hline Others & 0.2 & 0.117 & $0.02-1.6$ & 0.7 & 0.783 & $0.0-11.2$ \\
\hline \multicolumn{7}{|l|}{ Intervention ${ }^{4}$} \\
\hline Clip & 3.0 & 0.067 & $0.9-9.4$ & 4.9 & 0.082 & $0.8-28.9$ \\
\hline Fisher Scale $>$ II $^{5}$ & 2.3 & 0.246 & $0.6-8.9$ & 3.5 & 0.217 & $0.5-25.5$ \\
\hline WFNS grade $>$ III $^{6}$ & 2.6 & 0.116 & $0.8-8.7$ & 1.1 & 0.938 & $0.1-9.9$ \\
\hline APACHE subscore $>15^{7}$ & 7.4 & 0.016 & $1.5-37.7$ & 26.4 & 0.025 & $1.5-465.7$ \\
\hline \multicolumn{7}{|c|}{$\begin{array}{l}\text { ACOMM, anterior communicating artery; OR, odds ratio; CI, confidence interval; FT7, took the drug by feeding tube in } \\
\text { the first week; WFNS, World Federation of Neurological Surgeons; MCA, middle cerebral artery; PCOM, posterion } \\
\text { communicating artery. }{ }^{1} \text {, compared to whole tablets; }{ }^{2} \text {, compared to those who are not heavy alcohol drinkers; }{ }^{3} \text {, compared } \\
\text { to MCA aneurysm location as a reference; }{ }^{4}, \text { compared to endovascular coiling as a reference; }{ }^{5} \text {, compared to those with } \\
\text { Fisher Scale } \leq \mathrm{II} ;{ }^{6}, \text { compared to those with WFNS grade } \leq \mathrm{III} ;{ }^{7} \text {, compared to those with APACHE subscore } \leq 15 \text {. A total } \\
\text { of } 74 \text { patients were included in the multivariate analysis ( } 6 \text { patients excluded due to missing angiography report; } 2 \text { missing } \\
\text { Fisher scale and } 3 \text { had other interventions and excluded by STATA). }\end{array}$} \\
\hline
\end{tabular}

\section{The impact of nimodipine administration through enteral feeding tube over 21 days on patient outcomes}

As a secondary aim, the impact of nimodipine administration techniques over the 21-days period on angiographic vasospasm, DCI and hospital mortality was tested using logistic regression modeling. Nimodipine administration by feeding tube was not significantly associated with angiographic vasospasm or mortality over 21 days in patients with SAH. However, among the 58 patients with Fisher scale III-IV, nimodipine administration by feeding tube over the 21-days period was independently associated with DCI (OR 38.1 compared to those receiving whole tablets, 95\% CI 1.4-1067.9, $p$ value 0.032; ROC AUC 0.94, HL-test not significant) as shown in Table 4. 
Table 4. Crude and adjusted odds ratios of variables included in the final multivariate logistic regression model of DCI over 21 days (Fisher I-II was dropped from analysis).

\begin{tabular}{|c|c|c|c|c|c|c|}
\hline & \multicolumn{3}{|c|}{ Univariate Regression } & \multicolumn{3}{|c|}{ Multivariate Regression } \\
\hline & OR & $p$ value & $95 \% \mathrm{CI}$ & OR & $p$ value & $95 \% \mathrm{CI}$ \\
\hline Nimodipine (FT21) 1 & 5.4 & 0.066 & $0.9-33.0$ & 38.1 & 0.032 & $1.4-1067.9$ \\
\hline $\mathrm{Age}^{2}$ & 1.0 & 0.199 & $0.9-1.0$ & 0.9 & 0.193 & $0.8-1.0$ \\
\hline $\mathbf{B M I}^{2}$ & 1.1 & 0.027 & $1.01-1.3$ & 1.2 & 0.157 & $0.9-1.4$ \\
\hline \multicolumn{7}{|l|}{ Smoking $^{3}$} \\
\hline Non smoker & Ref & 0.154 & $0.0-2.0$ & Ref & 0.334 & $0.0-9.1$ \\
\hline Smoker/Ex-smoker & 0.2 & 0.917 & $0.1-6.5$ & 0.1 & 0.755 & $0.0-17.7$ \\
\hline Unknown & 0.9 & & & 0.6 & & \\
\hline WFNS grade $>$ III $^{4}$ & 1.0 & 0.975 & $0.2-6.2$ & 0.2 & 0.351 & $0.0-7.1$ \\
\hline APACHE II subscore $>15^{5}$ & 8.2 & 0.046 & $1.04-64.0$ & 18.4 & 0.174 & $0.3-1226.4$ \\
\hline \multicolumn{7}{|c|}{$\begin{array}{l}\text { DCI, delayed cerebral ischemia ; OR, odds ratio; CI, confidence interval; FT21, took the drug by feeding tube over } 21 \\
\text { days period; BMI, body mass index; WFNS, World Federation of Neurological Surgeons; APACHE II, Acute Physiology } \\
\text { and Chronic Health Evaluation. ' }, \text { compared to whole tablets; }{ }^{2} \text {, age and BMI are continous variables; }{ }^{3} \text {, compared to non- } \\
\text { smokers as a reference; }{ }^{4} \text {, compared to those with WFNS grade } \leq \text { III; }{ }^{5} \text {, compared to those with APACHE II subscore } \leq \\
\text { 15. A total of } 57 \text { patients were included in the multivariate analysis ( } 1 \text { patients excluded due to missing BMI value). }\end{array}$} \\
\hline
\end{tabular}

\section{DISCUSSION}

In the present study, nimodipine mode of administration was associated with vasospasm in the first week from SAH onset where patients receiving nimodipine via FT had increased odds of moderate to severe angiographic vasospasm compared to those administered it as whole tablets. Furthermore, patients who received nimodipine via feeding tube over the 21 days period were found to have increased odds of DCI compared to those administered whole tablets. To our knowledge, this is the first report suggesting an association between nimodipine technique of oral administration and vasospasm and DCI.

In our institution, all patients presenting with SAH receive oral nimodipine $60 \mathrm{mg}$ every 4 hours for 21 days or until discharge whichever comes first. Patients who have altered mental status, mechanically ventilated or unable to swallow are likely to get nimodipine tablets crushed at bedside and administered immediately through enteral feeding tubes (FT) such as nasogastric or orogastric tubes. The observed associations between nimodipine administration technique and vasospasm and DCI could be attributed to the reduced systemic exposure to nimodipine secondary to reduced oral bioavailability in those getting nimodipine vis feeding tubes.. The oral bioavailability of nimodipine has been reported to range from 3 to $30 \%$ with time to peak concentration ranging from $0.5-1 \mathrm{~h}$ $(17,18)$. Few studies have reported the reduced bioavailability of nimodipine administered through
FT in those with high grade $\mathrm{SAH}(12,13,19)$. Abboud et al have conducted a pharmacokinetic study on nimodipine enteral administration in SAH patients. In patients who were unable to swallow, the tablets were crushed and administered through nasogastric tubes. They have reported that nimodipine exposure as measured by the area under the concentration-time curve (AUC) in the FT group was lower than those who swallowed the whole tablets (median AUC 23.1 vs. $52.3 \mathrm{ng} . \mathrm{h} / \mathrm{ml}$, respectively, $p$ value 0.006 ). In addition, two high grade patients had undetectable plasma levels of nimodipine (13). Similarly, Kumana et al. have reported reduced bioavailability of nimodipine in a patient given crushed tablets through gastric tube (12). Also, Soppi et al have reported lower plasma levels of nimodipine in 3 patients with high grade SAH who were given nimodipine extemporaneously prepared suspension through nasogastric tube (19). None of those studies, however, has reported a correlation between reduced exposure and patient outcomes. Riva et al. have reported an association between nimodipine cerebrospinal fluid concentrations and neurological outcomes at 9 months following SAH onset but they were unable to find such correlation with plasma concentrations (14). It should be noted, however, that all their patients were dosed using nimodipine IV infusion and their plasma concentrations ranged from 24.9 to $71.8 \mathrm{ng} / \mathrm{ml}$, concentrations way above what has been reported in patients given oral dosing $(17,19)$. Taken together, although the evidence supporting a correlation between nimodipine concentrations and 
patient outcomes is scarce and not clear, lack of absorption definitely denies the benefits of any drug used systemically suggesting the need for an alternate formulation or route of administration. Oral nimodipine is also available in other countries as oral capsule and oral suspension. However, it is not clear if the observed reduced bioavailability is a function of the formulation itself, altered pharmacokinetics in SAH patients with high disease severity or both. Reduced bioavailability of drugs secondary to gastrointestinal dysfunction in critically ill patients and those in pain has been reported previously (20, 21). This could explain, at least in part, the reported reduced bioavailability of nimodipine in sicker patients. Intravenous nimodipine was compared to the oral route in two small randomized trials $(22,23)$. Both studies have found no difference in patient outcomes; however, the number of patients with high Hunt and Hess grade (IV and V) was small to draw conclusions on the comparability of both routes in high grade patients. Further research is needed.

In the present study, patients who received nimodipine via FT had higher incidence of moderate and severe angiographic vasospasm compared to those who received nimodipine as a whole tablet. On the other hand, most of the randomized controlled trials reporting the benefits of nimodipine on functional outcomes did not show a difference in vasospasm between nimodipine and placebo treated cohorts (6-9). The reason for this discrepancy is unclear and warrants further investigation especially many of the landmark trials did not include high grade patients (Hunt and Hess grades IV and V) where nimodipine is generally given via FT (24). In addition, nimodipine benefit was not demonstrated in patients with Hunt and Hess grade 5 (9). Approximately $7 \%$ of our cohort have developed DCI which is lower than what is reported in literature (6-9). This could be attributed to the retrospective chart review design where criteria for DCI may not have been documented in the patient's paper chart underestimating the incidence of DCI. However, each patient has been discussed among authors to confirm the presence or absence of DCI. Despite the reduced prevalence of DCI in our study, FT administration of nimodipine over the 21 days period were associated with DCI.

Our study has limitations. The main limitation is confounding by indication. The group of patients who got nimodipine via FT are generally sicker (higher WFNS grade, Fisher scale and APACHE II subscore), which could have contributed to the observed findings. In addition, the FT group were more likely to get surgical clipping than endovascular coiling further increasing the risk for complications. However, after controlling for those confounders, the association between nimodipine FT administration and worse outcomes still existed, highlighting the need for further investigation. Furthermore, due to the retrospective nature of our study, we were unable to compare the functional outcomes following hospital discharge. A prospective study is recommended to determine if FT administration is an independent predictor to poor functional outcomes. In addition, the retrospective design is prone to bias, missing data and confounding. Although we controlled for all possible confounders, we may have not controlled for unknown confounders that may have biased our results. This is a single centre study with small sample size which may limit the generalizability of the findings; however, it warrants conducting a multicentre study to confirm the study findings.

\section{CONCLUSION}

Our findings suggest that nimodipine administration via enteral feeding tubes may be associated with vasospasm and DCI in subarachnoid hemorrhage patients secondary to reduced exposure. Prospective studies are needed to confirm such association and alternate methods of administration should be explored to ensure patients are getting the benefits of nimodipine.

\section{FUNDING}

There is no funding associated with this work

\section{CONFLICTS OF INTEREST}

The authors declare that they have no conflicts of interest

\section{REFERENCES}

1. Connolly ES, Jr., Rabinstein AA, Carhuapoma JR, Derdeyn CP, Dion J, Higashida RT, et al. Guidelines for the management of aneurysmal subarachnoid hemorrhage: a guideline for healthcare professionals from the American Heart Association/american Stroke Association. Stroke. 2012;43(6):1711-37. 
2. Suarez JI, Tarr RW, Selman WR. Aneurysmal subarachnoid hemorrhage. N Engl J Med. 2006;354(4):387-96.

3. Diringer MN, Bleck TP, Claude Hemphill J, 3rd, Menon D, Shutter L, Vespa P, et al. Critical care management of patients following aneurysmal subarachnoid hemorrhage: recommendations from the Neurocritical Care Society's Multidisciplinary Consensus Conference. Neurocrit Care. 2011;15(2):211-40.

4. Francoeur CL, Mayer SA. Management of delayed cerebral ischemia after subarachnoid hemorrhage. Crit Care. 2016;20(1):277.

5. Frontera JA, Fernandez A, Schmidt JM, Claassen J, Wartenberg KE, Badjatia $\mathrm{N}$, et al. Defining vasospasm after subarachnoid hemorrhage: what is the most clinically relevant definition? Stroke. 2009;40(6):1963-8.

6. Philippon J, Grob R, Dagreou F, Guggiari M, Rivierez M, Viars P. Acta Neurochir (Wien) (1986) 82:110-114 eCt ahirurgica Prevention of Vasospasm in Subarachnoid Haemorrhage. A Controlled Study with Nimodipine. 1986:110-4.

7. Pickard JD, Murray GD, Illingworth R, Shaw MD, Teasdale GM, Foy PM, et al. Effect of oral nimodipine on cerebral infarction and outcome after subarachnoid haemorrhage: British aneurysm nimodipine trial. BMJ. 1989;298(6674):636-42.

8. Allen GS, Ahn HS, Preziosi TJ, Battye R, Boone SC, Boone SC, et al. Cerebral arterial spasm--a controlled trial of nimodipine in patients with subarachnoid hemorrhage. N Engl J Med. 1983;308(11):619-24.

9. Petruk KC, West M, Mohr G, Weir BK, Benoit BG, Gentili F, et al. Nimodipine treatment in poor-grade aneurysm patients. Results of a multicenter doubleblind placebo-controlled trial. J Neurosurg. 1988;68(4):505-17.

10. Connolly ES, Rabinstein AA, Carhuapoma JR, Derdeyn CP, Dion J, Higashida RT, et al. Guidelines for the management of aneurysmal subarachnoid hemorrhage: A guideline for healthcare professionals from the american heart association/american stroke association. Stroke. 2012;43(6):1711-37.

11. NIMOTOP(R) Product Monograph, Mississauga, Ontario. Bayer Inc.; 2014.

12. Kumana CR, Kou M, Yu YL, Fong KY, Fung CF, Chang $\mathrm{CM}$, et al. Investigation of nimodipine pharmacokinetics in Chinese patients with acute subarachnoid haemorrhage. European journal of clinical pharmacology. 1993;45(4):363-6.

13. Abboud T, Andresen H, Koeppen J, Czorlich P, Duehrsen L, Stenzig J, et al. Serum levels of nimodipine in enteral and parenteral administration in patients with aneurysmal subarachnoid hemorrhage. Acta Neurochirurgica. 2015;157(5):763-7.

14. Riva R, Pegoli M, Contin M, Perrone A, Mohamed S, Zanello M. Cerebrospinal Fluid Concentrations of
Nimodipine Correlate With Long-term Outcome in Aneurysmal Subarachnoid Hemorrhage: Pilot Study. Clin Neuropharmacol. 2019.

15. Harris PA, Taylor R, Thielke R, Payne J, Gonzalez N, Conde JG. Research electronic data capture (REDCap)--a metadata-driven methodology and workflow process for providing translational research informatics support. J Biomed Inform. 2009;42(2):377-81.

16. Vergouwen MD, Vermeulen M, van Gijn J, Rinkel GJ, Wijdicks EF, Muizelaar JP, et al. Definition of delayed cerebral ischemia after aneurysmal subarachnoid hemorrhage as an outcome event in clinical trials and observational studies: proposal of a multidisciplinary research group. Stroke. 2010;41(10):2391-5.

17. Langley MS, Sorkin EM. Nimodipine: A review of its pharmacodynamic and pharmacokinetic properties, and therapeutic potential in cerebrovascular disease. Drugs. 1989;37(5):669-99.

18. Muck W, Ahr G, Kuhlmann J. Nimodipine. Potential for drug-drug interactions in the elderly. Drugs \& aging. 1995;6(3):229-42.

19. Soppi V, Kokki H, Koivisto T, Lehtonen M, HelinTanninen M, Lehtola $S$, et al. Early-phase pharmacokinetics of enteral and parenteral nimodipine in patients with acute subarachnoid haemorrhage - a pilot study. European Journal of Clinical Pharmacology. 2007;63:355-61.

20. Boucher BA, Wood GC, Swanson JM. Pharmacokinetic changes in critical illness. Crit Care Clin. 2006;22(2):255-71, vi.

21. Jamali F, Kunz-Dober CM. Pain-mediated altered absorption and metabolism of ibuprofen: an explanation for decreased serum enantiomer concentration after dental surgery. $\mathrm{Br} \mathrm{J}$ Clin Pharmacol. 1999;47(4):391-6.

22. Soppi V, Karamanakos PN, Koivisto T, Kurki MI, Vanninen R, Jaaskelainen JE, et al. A randomized outcome study of enteral versus intravenous nimodipine in 171 patients after acute aneurysmal subarachnoid hemorrhage. World Neurosurg. 2012;78(1-2):101-9.

23. Kronvall E, Undren P, Romner B, Saveland H, Cronqvist M, Nilsson OG. Nimodipine in aneurysmal subarachnoid hemorrhage: a randomized study of intravenous or peroral administration. J Neurosurg. 2009;110(1):58-63.

24. Dorhout Mees SM, Rinkel GJ, Feigin VL, Algra A, van den Bergh WM, Vermeulen M, et al. Calcium antagonists for aneurysmal subarachnoid haemorrhage. Cochrane Database Syst Rev. 2007(3):CD000277. 